\title{
An efficient soft de-mapping algorithm for APSK signals
}

\author{
Wei You ${ }^{1,}$, , Daoxing Guo ${ }^{1, a}$ and $X \mathrm{Y} \mathrm{Yi}^{1, \mathrm{a}}$ \\ ${ }^{1}$ College of Communications Engineering, PLA University of Science and Technology, Nanjing \\ 210007, China \\ aemail: youwin258@sina.com
}

Keywords: soft de-mapping; APSK; LDPC code; iterative decoding; hard decision

Abstract: In this paper, we present an efficient soft de-mapping method for M-ary APSK modulation based on hard decision thresholds (HDT), which significantly reduces the computational complexity compared with the maximum likelihood detection (MLD). Furthermore, bit error rate (BER) performance of several soft de-mapping methods combined with LDPC codes are provided. Simulation results reveal that the proposed method largely reduces the computational complexity with nearly the same performance to MLD.

\section{INTRODUCTION}

The standard of Digital Video Broadcasting via Satellite-Second Generation (DVB-S2) was proposed by the European Telecommunications Standards Institute (ETSI), which contains a number of advanced technologies to increase the quality of service (QoS) for satellite communications, for instance, adaptive coding and modulation combing with APSK signals. Regarding channel encoding, DVB-S2 standard adopts the concatenated coding mechanism that exploits $\mathrm{BCH}$ code as the outer code and the LDPC code as inner code to improve the error correcting performance. Compared with the previous DVB-S standard, DVB-S2 is capable of improving the capacity by $30 \%$. Since accurate soft decision bit information (SDBI) is one of the inputs for the decoder, it plays an important role to achieve good decoding performance.

As is well known that maximum likelihood detection (MLD) is able to obtain the most accurate SDBI in theory, however, the computational complexity boosts with the increase of the modulation order. To handle with this problem, many efforts have been devoted to focus on reducing the computational complexity. In [3]-[5], the log likelihood ratio(LLR) is calculated by reducing the constellation point, while in [6] and [7] the hard decision threshold value (HDT) was proposed to calculate SDBI for QPSK and 16QAM, respectively.

Motivated by these observations, we extend the method of HDT to the M-APSK signal, and thus an efficient novel de-mapping algorithm based on HDT is proposed, which significantly decreases the computational complexity of the decoder. Simulation results demonstrate the effectiveness of our proposed algorithm.

\section{MLD algorithm for LLR calculation}

To begin with, we present the posteriori LLR of each receiving bit for MLD algorithm. Assuming that the transmitter emits 0 and 1 with equal probability, i.e., $P\left(b_{i}=0\right)=P\left(b_{i}=1\right)=1 / 2$, and thus the LLR of MLD is given by:

$$
L\left(b_{i} \mid y\right)=\log \left(\sum_{s: b_{i}(i)=1} \exp \left(-\frac{1}{2 \sigma^{2}}(y-s)^{2}\right) / \sum_{s: b_{i}(i)=0} \exp \left(-\frac{1}{2 \sigma^{2}}(y-s)^{2}\right)\right)
$$

where $s$ denotes the scatterplot of M-APSK symbol, which consists of $m=\log _{2} M$ bits; $\sigma^{2}$ denotes the variance of additive Gauss white noise (AWGN); $y$ is the receiving symbol. Based on the equation above, we find that the computational complexity of the LLR has an exponential growth with the increase of $m$, especially for high order modulations. An approximation approach is to replace the summation in (1) by the largest one, i.e., 


$$
L\left(b_{i} \mid y\right) \approx \log \left(\max _{s \cdot b_{i}(i)=1} \exp \left(-\frac{1}{2 \sigma^{2}}(y-s)^{2}\right) / \max _{s: b_{i}(i)=0} \exp \left(-\frac{1}{2 \sigma^{2}}(y-s)^{2}\right)\right)
$$

which reduces to Max LLR algorithm. Therefore, we have:

$$
L\left(b_{i} \mid y\right) \approx \frac{1}{2 \sigma^{2}}\left[\min _{s: b_{i}(i)=0}(y-s)^{2}-\min _{s: b_{i}(i)=1}(y-s)^{2}\right]
$$

\section{HDT based soft de-mapping algorithm}

a) The origin of HDT algorithm

Let us take BPSK modulation for instance, the receiving symbol +1 and -1 represent bit 1 and bit 0 , respectively. Thus the LLR of MLD is given by

$$
L(b \mid y)=\log \left(\exp \left(-\frac{1}{2 \sigma^{2}}(y-1)^{2}\right) / \exp \left(-\frac{1}{2 \sigma^{2}}(y+1)^{2}\right)\right)
$$

By dividing the nominator and denominator with $\exp \left(-(y+1)^{2} / 2 \sigma^{2}\right)$, simutaneously, we attain

$$
L(b \mid y)=\log \left(\exp \left(4 y /\left(2 \sigma^{2}\right)\right)\right)=2 y / \sigma^{2}
$$

To make the analysis more tractable, here we denote $p=2 / \sigma^{2}$, then the expression can be re-expressed by

$$
L(b \mid y)=p y
$$

Based on (6), it reveals that the calculation of $L(b \mid y)$ is equal to use the coefficient $p$ to multiply the symbol, which is similar for QPSK and 16QAM signal [8]-[10].

b) 16-APSK Signal

Different from the modulation mode aforementioned, APSK modulation does not require strict Gray code mapping, and the ratio of each radius is not always an integer, which increases the difficulty of designing APSK signals. Fig. 1 shows the constellation diagram of 16-APSK, $b_{i}$ is the $i$-th bit of each symbol and $b_{i}, i \in\{1234\}$. We compute SDBI by the above idea, i.e., $L\left(b_{i} \mid y\right)=p \hat{b}_{i}(y)$. In the following, we will give the calculation method of $\hat{b}_{i}(y)$. From the constellation diagram, we know that $b_{4}$ and $b_{2}$ distributes symmetrically by the horizontal axis and vertical axis, respectively and we can get $\hat{b}_{4}(y)=-I(y), \hat{b}_{3}(y)=-R(y)$, where $I(y)$ indicates the in-phase component and $R(y)$ indicates the quadrature component. Taking the same method as 16QAM to calculate $b_{2}$ and $b_{1}$, we achieve that $\hat{b}_{2}(y)=L_{2}-|I(y)|, \hat{b}_{1}(y)=L_{1}-|R(y)|$. Where $L_{1}=\sum_{i=0}^{3} R\left(s_{i}\right) / 4, L_{2}=\sum_{i=0}^{3} I\left(s_{i}\right) / 4$, it is easily known that $L_{1}=L_{2}$ by the symmetrical characteristic.

c) 32-APSK signal

The $\hat{b}_{i}(y)$ of 32 -APSK is determined by the following formula:

$$
L\left(b_{1} \mid y\right)=\left\{\begin{array}{cc}
\left(T_{1}-|I(y)|\right) & |I(y)|<T_{1} \\
p \max \left\{-y-r_{d 2} e^{j \frac{4 \pi}{16}}, T_{1}-|I(y)|\right\} & |I(y)| \geq T_{1} \& \& y<r_{d 2} \& \& \theta<4 \pi / 16 \\
p \max \left\{T_{1}-|I(y)|, y-r_{d 2}\right\} & |I(y)| \geq T_{1} \& \& y<r_{d 2} \& \& 4 \pi / 16<\theta<6 \pi / 16 \\
p \max \left\{-y-r_{d 2} e^{j \frac{6 \pi}{16}}, T_{1}-|I(y)|\right\} & |I(y)| \geq T_{1} \& \& y<r_{d 2} \& \& \theta \geq 6 \pi / 16 \\
p \max \left\{T_{1}-|I(y)|, y \sin (\theta-4 \pi / 16)\right\} & |I(y)| \geq T_{1} \& \& y \geq r_{d 2} \& \& \theta<4 \pi / 16 \\
p \min \left\{y \sin (\theta-4 \pi / 16), y-r_{d 2}, y \sin (6 \pi / 16-\theta)\right\} & |I(y)| \geq T_{1} \& \& y \geq r_{d 2} \& \& 4 \pi / 16<\theta<6 \pi / 16 \\
p y \sin (6 \pi / 16-\theta) & |I(y)| \geq T_{1} \& \& y \geq r_{d 2} \& \& \theta \geq 6 \pi / 16 \\
L\left(b_{2} \mid y\right)=p\left(y-r_{d 1}\right) & \\
L\left(b_{3} \mid y\right)=-p R(y) & \\
L\left(b_{4} \mid y\right)=-p I(y) &
\end{array}\right.
$$




$$
L\left(b_{5} \mid y\right)=\left\{\begin{array}{lc}
p\left(T_{5}-|R(y)|\right) & \text { if } y<r_{d 2} \\
p y \sin \left(\theta-\frac{4 \pi}{16}\right) & \text { else }
\end{array}\right.
$$

where $T_{1}=\left(\sum_{i=0}^{5} I\left(s_{i}\right)\right) / 6, T_{5}=\left(\sum_{i=0}^{3} R\left(s_{i}\right)\right) / 4, \quad r_{d 1}=\left(r_{1}+r_{2}\right) / 2, \quad r_{d 2}=\left(r_{2}+r_{3}\right) / 2$ and,$\theta=\tan ^{-1}(|I(y)| /|R(y)|)$.

Fig. 2 to 4 depict the constellation diagram and the HDT of each bit, respectively.
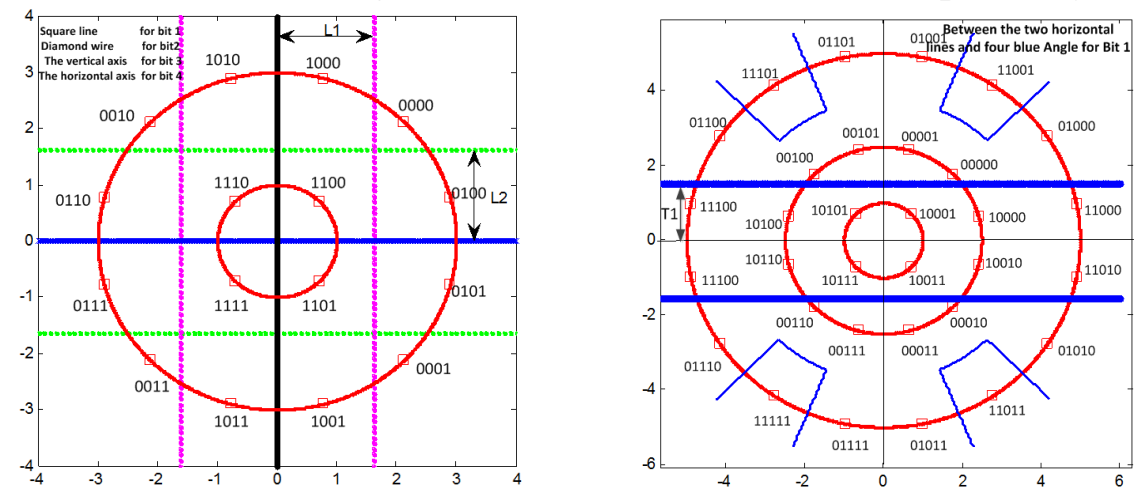

Fig. 1 The HDT of 16APSK for each bit.

Fig. 2 The HDT of 32APSK for bit 1.
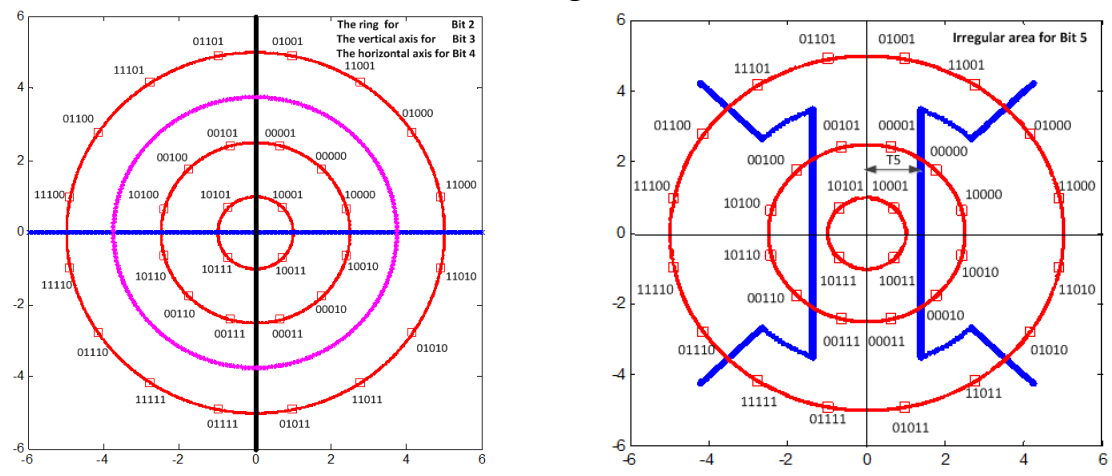

Fig. 3 The HDT of 32APSK for bit 234 Fig. 4 The HDT of 32APSK for bit 5

\section{SIMULATIONS AND DISCUSSION}

In this section, the performance of LLR, MaxLLR and HDT algorithms is investigated by Monte Carlo simulations. Notably, each block of data consists of 1920 bits and LDPC code is applied with code rate $1 / 2$ which is the standard code in IEEE 802.16e. We use the BP algorithm for decoding and the number of iteration is set as 50.. From the simulation results, we find that the performance of the proposed algorithm is very close to the performance of LLR and MaxLLR. We set the BER of $10^{-5}$, the HDT algorithm needs more about $0.3 \mathrm{~dB}$ than LLR algorithm and $0.2 \mathrm{~dB}$ than MaxLLR algorithm.
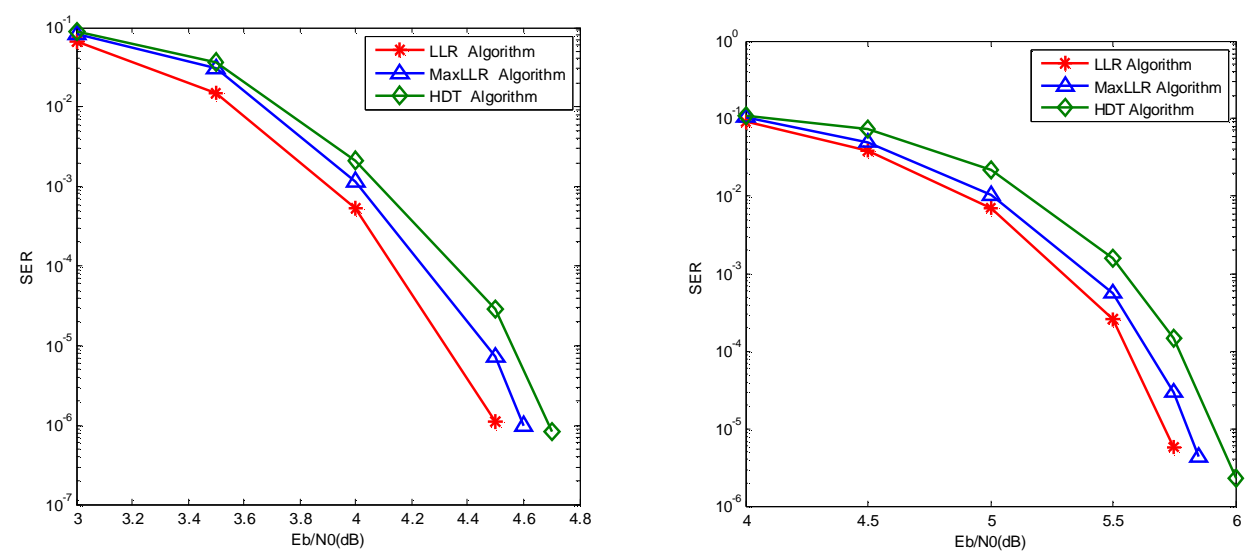

Fig. 5 The BER curves for 16-APSK

Fig. 6 The SER curves for 32-APSK 
The computation complexity of the considered algorithms is compared in the following table. From the table below, we find that the proposed algorithm has greatly reduced the amount of computation complexity.

Table 1 The computational complexity comparison.

\begin{tabular}{|c|c|c|c|c|c|c|}
\hline & \multicolumn{2}{|c|}{ ML } & \multicolumn{2}{c|}{ MaxLLR } & \multicolumn{2}{c|}{ HDT } \\
\hline & $16-A P S K$ & $32-A P S K$ & $16-A P S K$ & $32-A P S K$ & $16-A P S K$ & $32-A P S K$ \\
\hline$|\cdot|^{2}$ & 16 & 32 & 16 & 32 & 4 & 11 \\
\hline $\exp ()$ & 16 & 32 & 0 & 0 & 0 & 0 \\
\hline $\log ()$ & 4 & 5 & 0 & 0 & 0 & 0 \\
\hline COMP & 0 & 0 & 64 & 160 & 0 & 7 \\
\hline
\end{tabular}

\section{CONCLUSIONS}

This paper presents an efficient soft de-mapping method for M-ary APSK based on hard decision thresholds (HDT), which significantly reduces the computational complexity compared with maximum likelihood detection (MLD). Furthermore, the loss of bit error rate (BER) performance is limited to a very small range. Simulation results demonstrate the effectiveness of our proposed method.

\section{REFERENCES}

[1] ETSI EN 302307 v1.2.1. Second Generation Framing Structure, Channel Coding and Modulation Systems for Broadcasting, Interactive Services, News Gathering and other Broadband Satellite Applications(DVB-S2), Final draft, 2009.

[2] W. Gappmair, J. Holzleitner, "Detector characteristic for decision directed carrier phase recovery of 16/32-APSK signals," IEEE Electronics Letters, vol. 42, no. 25, pp. 1464 - 1466, 2006.

[3] C.D. Ryu, J.W. Park, M.H. Sunwoo. Simplified soft-decision de-mapping algorithm for digital video broadcasting system[J]. Electronics Letters,2010,46(12):840-842.

[4] E.Yao, S.Yang, W. Jiang. A Simplified Soft Decision De-mapping Algorithm of 16-APSK Signals in AWGN Channel[J]. Proceedings of COSNWCC 2010,vol.1,pp.103-106

[5] K.K. Cho, K.M. Hyun, D.W. Yoon, etc. An Approximated Soft Decoding Algorithm of 16-APSK Signal for DVB-S2[J]. Proceedings of ICCE 2007,pp.1-2.

[6] F. Tosato, P. Bisaglia. Simplified soft-output de-mapper for binary interleaved COFDM with application to HIPERLAN/2[J].Proceedings of IEEE ICC 2002,vol.2,pp.664-668

[7] E.G. Larsson, J.Jalden. Fixed Complexity Soft MIMO Detection via Partial Marginalization[J]. IEEE Transactions on Signal Processing, vol.56, pp.3397-3407, 2008.

[8] R.D. Gaudenzi, A. Guillen, A. Martinez. Performance analysis of turbo-coded APSK modulations over nonlinear satellite channels[J]. IEEE Trans on Wireless Commu, 2006,5(9):2396-2407

[9] ETSI EN 302307 v1.2.1. Second Generation Framing Structure, Channel Coding and Modulation Systems for Broadcasting, Interactive Services, News Gathering and other Broadband Satellite Applications (DVB-S2)[A].Final draft, 2009.

[10] W. Gappmair, J. Holzleitner. Detector characteristic for decision directed carrier phase recovery of 16/32-APSK signals[J]. IEEE Electronics Letters, vol. 42, no. 25, pp. 1464 - 1466, 2006. 